CTP TAMU-39/99

UPR-861-T

hep-th/9909184

September 1999

\title{
Supercharges, Killing Spinors and Intersecting Gauge Five-branes
}

\author{
E. Lima ${ }^{\dagger}$, H. Lü ${ }^{\dagger 1}$, B.A. Ovrut ${ }^{\dagger 2}$ and C.N. Pope ${ }^{\ddagger 3}$ \\ ${ }^{\dagger}$ Department of Physics and Astronomy \\ University of Pennsylvania, Philadelphia, Pennsylvania 19104 \\ ${ }^{\ddagger}$ Center for Theoretical Physics \\ Texas AEM University, College Station, Texas 77843
}

\begin{abstract}
$\underline{\text { ABSTRACT }}$
We obtain new solutions where a string and a pp-wave lie in the common worldvolume directions of the non-standard intersection of two gauge 5-branes in the heterotic string. The two 5-branes are supported by independent $S U(2)$ Yang-Mills instantons in their respective (non-overlapping) transverse spaces. We present a detailed study of the unbroken supersymmetry, focusing especially on a comparison between a direct construction of Killing spinors and a counting of zero eigenvalues in the annticommutator of supercharges. The results are in agreement with some previous arguments, to the effect that additional zero eigenvalues resulting from a "fine-tuning" between positive-energy and negative-energy contributions from different components in an intersection are spurious, and should not be taken as an indication of supersymmetry enhancements. These observations have a general applicability that goes beyond the specific example we study in this paper.
\end{abstract}

\footnotetext{
${ }^{1}$ Research supported in part by DOE grant DE-FG02-95ER40893

${ }^{2}$ Research supported in part by DOE grant DE-AC02-76ER03071

${ }^{3}$ Research supported in part by DOE Grant DE-FG03-95ER40917.
} 


\section{Introduction}

Over the years, a large menagerie of BPS $p$-brane solutions of the various supergravities has been discovered. These include the basic half-supersymmetric solutions such as the string [1] and 5-brane [2] in $D=10$, and the membrane [3] and 5-brane [4] in $D=11$. In addition, there are BPS solutions that preserve smaller fractions of supersymmetry, which admit an interpretation as intersections [5] of the basic half-supersymmetric building blocks.

The intersections themselves can be divided into two broad categories. Firstly, there are the "standard" intersections, which can all be interpreted, by means of a toroidal reduction to some sufficiently low dimension, as multi-charge $p$-branes for some single specific $p$, where the charges are carried by different field strengths of the lower-dimensional theory. The $p$-brane for each charge species by itself oxidises back to one specific component of the intersection in the higher dimension, with the full set of intersecting objects arising when all the different charge species are turned on. This draws attention to the fact that one should also include BPS configurations that go somewhat beyond what one would normally call a "p-brane." If the charge-carrying field in the lower dimension is a Kaluza-Klein vector, then back in the original higher dimension it will have become part of the off-diagonal structure of the internal part of the metric. If the Kaluza-Klein vector carried an electric charge in the lower dimension, this will give rise to a higher-dimensional metric with a pp-wave propagating along one of the internal directions. If, on the other hand, the Kaluza-Klein field carried a magnetic charge in the lower dimension, then, from the higher-dimensional standpoint, the metric will have a Taub-NUT-like monopole structure, sometimes simply called a "NUT" for short. Various p-brane examples can be found in [6] and references therein. Four-dimensional black hole solutions were classified in [7]. The intersection rules for $p$-branes in $D=11$ and $D=10$ were classified in [8]. The classification of $p$-branes and standard intersections in maximal supergravities for $2 \leq D \leq 11$ was given in [10, 11].

The second category of intersecting solutions consists of what may be called "nonstandard" intersections. These are examples where there is no simple lower-dimensional interpretation as a multi-charge $p$-brane. This is because in these solutions the harmonic functions for the intersecting ingredients are all independent of the overall transverse space. The first such example was constructed in [12], and further examples were studied in 13, 14, 8, 9]. A four-dimensional solution with three perpendicular intersecting membranes (domain walls in $D=4$ ) was constructed in [15], admitting an interpretation as a cosmological lattice universe model. In fact three is the maximal number of intersections (with all pair-wise intersections non-standard) that can occur in supergravity theories. The solutions that we 
shall be constructing in this paper involve a combination of standard and non-standard intersections.

Intersections in $D=10$ can arise in both the type II theories and also in the heterotic theory. Many of the solutions in the heterotic theory can also be viewed as solutions in the type II theories, since the subset of the latter that make use only of the NS-NS fields can be transferred across directly as solutions in the heterotic string. However, there are also further possibilities in the heterotic string, owing to the presence of the Yang-Mills fields. One possibility is to find solutions in which the Yang-Mills fields themselves carry charges that play a rôle in supporting the $p$-brane or intersection. However, in such circumstances one typically finds that the solution will not be a BPS one. Another possibility is to use the Yang-Mills fields to construct an instanton configuration in a four-dimensional transverse space, which can act as a non-singular source in place of the more customary point-charge singular sources in the harmonic functions describing the solution. One can think of the Yang-Mills fields here as playing the rôle of a "regulator," which smears out the pointcharge singularities. The first example of such an instanton-supported soliton was the "gauge 5-brane" constructed in [2]. A dyonic string in $D=6$, where both the electric and magnetic charges are supported by Yang-Mills instantons, was constructed in [16]. Such a configuration can also support a pp-wave propagating in the string world sheet [17.

The new solutions that we shall construct in this paper involve many of the ingredients mentioned above. Specifically, what we obtain is a ten-dimensional solution of the heterotic theory, describing the intersection of two 5-branes, a string, and a pp-wave. If the 5-brane charges are turned off, the string/wave intersection is of the "standard" type, which can be viewed as a 2-charge black hole in $D=9$, supported by the winding vector and the KaluzaKlein vector respectively. We shall first obtain the more general intersections, with the two 5-branes, a string and a pp-wave, as solutions using only the fields of the $N=1$ truncation of type II supergravities; in other words the metric, the 2-form potential and the dilaton. As usual in such solutions, there will be singular sources, corresponding to the locations of point charges or distributions of charges. Then, we shall show that we can generalise the solutions by using the Yang-Mills fields of the heterotic string to "smear out" the two sets of 5-brane charges. A novel feature, associated with the fact that the intersection between the two 5-branes is non-standard, is that we can introduce separate self-dual Yang-Mills instanton configurations in the two distinct transverse 4-spaces of the two 5-branes.

Having obtained the intersecting solutions, we then turn to a detailed discussion of their supersymmetry. There has been a rather confusing literature on the subject of the super- 
symmetry of multi-charge $p$-branes, and intersecting $p$-branes, and in the present paper we attempt to clarify some of these issues. Although our discussion will be focused on the particular case of interest here, it actually provides insights of a more widespread applicability. We shall be particularly concerned with addressing the issue of how one should interpret the occurrence of zero-eigenvalues of the matrix $\{Q, Q\}$ obtained by anticommuting the supercharges, and to what extent such zero eigenvalues can be taken as an indication of the corresponding existence of Killing spinors. In particular, when one calculates $\{Q, Q\}$ for BPS configurations involving more than one kind of charge species, one commonly finds that the number of zero-eigenvalues can become enhanced for particular fine tunings of the charges, by cancelling one contribution against another. This would appear to imply that the supersymmetry can be enhanced at these special charge values, leading even to preserved supersymmetry fractions such as $\frac{3}{4}$ or $\frac{7}{8}$ in some cases. Examples of this apparent phenomenon were found [18] and subsequently laid to rest [19] in the past. In this paper we examine the issue in the context of the new intersections of two 5-branes, a string and a pp-wave that we obtain here. We compare the results from the $\{Q, Q\}$ anticommutator with the results of direct computation of the Killing spinors, and we conclude again that the apparent "supersymmetry enhancements" suggested by the enlarged numbers of $\{Q, Q\}$ zero-eigenvalues at special charge values are spurious. The point is that the derivation of the connection between zero-eigenvalues of $\{Q, Q\}$ and the existence of Killing spinors involves certain assumptions about the global structure of the solutions, including the absence of naked singularities in the metric, and these assumptions are violated in all the cases where "fine-tuning" of charge parameters enlarges the number of zero-eigenvalues. (Some detailed discussion of this point was given in [21].) Thus our results here support the previous contention that no enhancements of supersymmetry occur at fine-tuned non-vanishing charge values.

The paper is organised as follows. In section 2 we construct the new solutions, comprising a non-standard intersection of two 5-branes together with a string and a pp-wave, within the framework of the $N=1$ truncation of type II supergravity. All the ingredients, including the 5-branes, have singular sources. Then, in section 3 , we generalise the solutions within the framework of the heterotic theory, by introducing self-dual Yang-Mills instantons to replace the singular 5-brane sources. In section 4 we examine the supersymmetry of the solutions, both from the type IIA or M-theoretic viewpoint and from the heterotic viewpoint. We show how the explicit results from solving the Killing-spinor equations compare

\footnotetext{
${ }^{1}$ As opposed to setting charges to zero, which obviously can enlarge the supersymmetry.
} 
with a counting of zero-eigenvalues in the anticommutator of supercharges, which clarifies the issue of when the zero-eigenvalue counting procedure gives trustworthy results for the determination of unbroken supersymmetry. In section 5, we discuss the near-horizon structure of the intersecting solutions. The paper ends with conclusions in section 6 .

\section{Intersections with singular sources}

In this section, we construct the new solution, which is a non-standard intersection of two 5-branes, a string and a pp-wave, within the framework of the $N=1$ truncation of the type II strings. Specifically, it is a solution of the theory described by the ten-dimensional Lagrangian

$$
\mathcal{L}_{10}=R * \mathbb{1}-\frac{1}{2} * d \phi \wedge d \phi-\frac{1}{2} e^{-\phi} * F_{(3)} \wedge F_{(3)},
$$

and is given by

$$
\begin{aligned}
d s_{10}^{2}= & K^{-3 / 4} H^{-1 / 4} \widetilde{H}^{-1 / 4}\left(-W^{-1} d t^{2}+W\left(d x+\eta_{w}\left(W^{-1}-1\right) d t\right)^{2}\right) \\
& +K^{1 / 4} H^{3 / 4} \widetilde{H}^{-1 / 4}\left(d y_{1}^{2}+\cdots+d y_{4}^{2}\right) \\
& +K^{1 / 4} H^{-1 / 4} \widetilde{H}^{3 / 4}\left(d z_{1}^{2}+\cdots+d z_{4}^{2}\right), \\
\phi= & -\frac{1}{2} \log [K /(H \widetilde{H})], \\
F_{(3)}= & \eta e^{\phi} *\left(\widetilde{H} d t \wedge d x \wedge d^{4} z \wedge d H^{-1}\right)+\tilde{\eta} e^{\phi} *\left(H d t \wedge d x \wedge d^{4} y \wedge d \widetilde{H}^{-1}\right) \\
& +\eta_{e} d t \wedge d x \wedge d K^{-1},
\end{aligned}
$$

where $H=H(\vec{y}), \widetilde{H}=\widetilde{H}(\vec{z}), K=K(\vec{y}, \vec{z})$ and $W=W(\vec{y}, \vec{z})$, and the ten coordinates have been split as $(t, x, \vec{y}, \vec{z})$ with $\vec{y}=\left(y_{1}, y_{2}, y_{3}, y_{4}\right)$ and $\vec{z}=\left(z_{1}, z_{2}, z_{3}, z_{4}\right)$. The quantities $\eta \tilde{\eta}, \eta_{e}$ and $\eta_{w}$ can each independently be chosen to be \pm 1 , giving a total of 16 equivalent solutions.2

We find that equations (2.2) give a solution provided that the functions $H$ and $\widetilde{H}$ are harmonic with respect to the flat spaces corresponding to their indicated coordinate dependences,

$$
\square_{\vec{y}} H=0, \quad \square_{\vec{z}} \widetilde{H}=0,
$$

while $H_{e}$ and $W$ satisfy the equations

$$
H^{-1} \square_{\vec{y}} K+\widetilde{H}^{-1} \square_{\vec{z}} K=0, \quad H^{-1} \square_{\vec{y}} W+\widetilde{H}^{-1} \square_{\vec{z}} W=0 .
$$

\footnotetext{
${ }^{2}$ These 16 solutions are all equivalent purely within the framework of the bosonic sector of the supergravity, but they are not all equivalent when supersymmetry is taken into account. This is because field strengths enter quadratically in the bosonic equations, but linearly in the supersymmetry transformation rules.
} 
The harmonic functions $H$ and $\widetilde{H}$ are associated with the two 5-branes in the intersection, $K$ is associated with the string, and $W$ with the pp-wave. Since there is no overlap between the coordinate dependences of the $H$ and $\widetilde{H}$ harmonic functions, it is evident that the intersection is of a non-standard type, since there is no lower dimension where the configuration could become a multi-charge $p$-brane. Furthermore, the functions $K$ and $W$ do not simply satisfy Laplace equations, but instead satisfy coupled equations that involve the 5-brane harmonic functions $H$ and $\widetilde{H}$. This also is a characteristic feature of non-standard intersections. Of course, we can find simple solutions for $K$ and $W$ by taking

$$
\begin{aligned}
K(\vec{y}, \vec{z}) & =\psi_{1}(\vec{y}) \psi_{2}(\vec{z})+\psi_{3}(\vec{y})+\psi_{4}(\vec{z}), \\
W(\vec{y}, \vec{z}) & =\chi_{1}(\vec{y}) \chi_{2}(\vec{z})+\chi_{3}(\vec{y})+\chi_{4}(\vec{z}),
\end{aligned}
$$

where the functions $\psi_{i}$ and $\chi_{i}$ are all harmonic in their respective subspaces. Note that solutions of this kind, but without the wave component (i.e. with $W=1$ ), were obtained previously in [22]. The solution can also be obtained from the dimensional reduction of the intersections of a wave, an M2-brane and two M5-branes in D=11, which were constructed in [23].

It is worth remarking that since the Lagrangian (2.1) can also be viewed as a consistent truncation of the type IIA theory, we can also regard the above solutions as originating from $D=11$. In fact we shall exploit this later, when we calculate the supersymmetry of the solutions.

\section{Instanton-supported intersections}

We shall now show that we can generalise the above intersection, by using the Yang-Mills fields of the heterotic string to provide instanton configurations that will allow the harmonic functions $H$ and $\widetilde{H}$ associated with the 5 -branes to be replaced by non-singular solutions in the heterotic string. A novel feature that arises here is that, owing to the non-overlapping nature of the 4-dimensional subspaces coordinatised by $\vec{y}$ and $\vec{z}$, where the functions $H(\vec{y})$ and $\widetilde{H}(\vec{z})$ find their support, we can introduce independent Yang-Mills instantons for the two 5-branes, using separate $S U(2)$ factors in the $E_{8} \times E_{8}$ or $S O(32)$ gauge group.

The low-energy effective action of the heterotic string is $N=1$ supergravity in $D=10$, coupled to $E_{8} \times E_{8}$ or $S O(32)$ Yang-Mills matter fields. We shall focus on two orthogonal $S U(2)$ subgroups of $E_{8} \times E_{8}$. The Lagrangian for the bosonic sector is given by

$$
\mathcal{L}_{10}=R * \mathbb{1}-\frac{1}{2} * d \phi \wedge d \phi-\frac{1}{2} e^{-\phi} * F_{(3)} \wedge F_{(3)}-\frac{1}{2} e^{-\frac{1}{2} \phi}\left(* G_{(2)}^{a} \wedge G_{(2)}^{a}+* \mathcal{G}_{(2)}^{\alpha} \wedge \mathcal{G}_{(2)}^{\alpha}\right),
$$


where the fields $G_{(2)}^{a}$ and $\mathcal{G}_{(2)}^{\alpha}$ are the Yang-Mills field strengths given by

$$
\begin{aligned}
G_{(2)}^{a} & =d B_{(1)}^{a}+\frac{1}{2} \epsilon^{a b c} B_{(1)}^{b} \wedge B_{(1)}^{c}, \\
\mathcal{G}_{(2)}^{\alpha} & =d \mathcal{B}_{(1)}^{\alpha}+\frac{1}{2} \epsilon^{\alpha \beta \gamma} \mathcal{B}_{(1)}^{\beta} \wedge \mathcal{B}_{(1)}^{\gamma},
\end{aligned}
$$

and $F_{(3)}$ is the three-form field strength, given by

$$
\begin{aligned}
F_{(3)}= & d A_{(2)}+\frac{1}{2} B_{(1)}^{a} \wedge d B_{(1)}^{a}+\frac{1}{6} \epsilon^{a b c} B_{(1)}^{a} \wedge B_{(1)}^{b} \wedge B_{(1)}^{c} \\
& +\frac{1}{2} \mathcal{B}_{(1)}^{\alpha} \wedge d \mathcal{B}_{(1)}^{\alpha}+\frac{1}{6} \epsilon^{\alpha \beta \gamma} \mathcal{B}_{(1)}^{\alpha} \wedge \mathcal{B}_{(1)}^{\beta} \wedge \mathcal{B}_{(1)}^{\gamma} .
\end{aligned}
$$

It satisfies the Bianchi identity

$$
d F_{(3)}=\frac{1}{2} G_{(2)}^{a} \wedge G_{(2)}^{a}+\frac{1}{2} \mathcal{G}_{(2)}^{\alpha} \wedge \mathcal{G}_{(2)}^{\alpha}
$$

We find that the Lagrangian (3.1) admits solutions of precisely the same form (2.2) as we obtained in the Introduction, describing the intersection of two 5-branes, a string and a pp-wave, except that now the functions $H, \widetilde{H}, K$ and $W$ satisfy the more general equations of motion

$$
\begin{aligned}
& \square_{\vec{y}} H=-\frac{1}{4} G_{i j}^{a} G_{i j}^{a}, \quad \square_{\vec{z}} \widetilde{H}=-\frac{1}{4} \mathcal{G}_{m n}^{\alpha} \mathcal{G}_{m n}^{\alpha}, \\
& \left(H^{-1} \square_{\vec{y}}+\widetilde{H}^{-1} \square_{z}\right) K=0, \quad\left(H^{-1} \square_{y}+\widetilde{H}^{-1} \square_{z}\right) W=0,
\end{aligned}
$$

where the index contractions in $G_{i j}^{a} G_{i j}^{a}$ and $\mathcal{G}_{m n}^{\alpha} \mathcal{G}_{m n}^{\alpha}$ are performed simply using the metrics $\delta_{i j}$ and $\delta_{m n}$ of the flat four-dimensional transverse spaces $d y^{i} d y^{i}$ and $d z^{m} d z^{m}$ respectively. The $S U(2)$ Yang-Mills fields $G_{(2)}^{a}$ and $\mathcal{G}_{(2)}^{\alpha}$ satisfy the self-duality equations $* G_{i j}^{a}=G_{i j}^{a}$ and $* \mathcal{G}_{m n}^{\alpha}=\mathcal{G}_{m n}^{\alpha}$ in the four-dimensional flat transverse spaces respectively, where $*$ denotes Hodge duality in these flat spaces. To be precise, we should remark that the bosonic equations can be satisfied by taking the Yang-Mills fields to be either self-dual or anti-selfdual (with independent such choices for the two $S U(2)$ factors). As with the signs in the expression for the 3-form field strength in (2.2), the different choices that one makes can impinge upon the supersymmetry of the solutions, as we shall see later.

The equations for $K$ and $W$ depend on $H$ and $\widetilde{H}$. In this paper, we shall focus on solutions where $K$ and $W$ are independent of $H$ and $\widetilde{H}$. Thus, as in (2.5), we may simply take solutions for $K$ and $W$ built from harmonic functions in the two subspaces:

$$
\begin{aligned}
K & =\left(1+\sum_{\alpha} \frac{2 Q_{e}^{\alpha}}{\left|\vec{y}-\vec{y}_{\alpha}\right|^{2}}\right)\left(1+\sum_{\alpha^{\prime}} \frac{2 Q_{e}^{\alpha^{\prime}}}{\left|\vec{z}-\vec{z}_{\alpha^{\prime}}\right|^{2}}\right)+\sum_{\alpha^{\prime \prime}} \frac{2 Q_{e}^{\alpha^{\prime \prime}}}{\left|\vec{y}-\vec{y}_{\alpha^{\prime \prime}}\right|^{2}}+\sum_{\alpha^{\prime \prime \prime}} \frac{2 Q_{e}^{\alpha^{\prime \prime \prime}}}{\left|\vec{y}-\vec{y}_{\alpha^{\prime \prime \prime}}\right|^{2}}, \\
W & =\left(1+\sum_{\beta} \frac{2 P_{w}^{\beta}}{\left|\vec{y}-\vec{y}_{\beta}\right|^{2}}\right)\left(1+\sum_{\beta^{\prime}} \frac{2 P_{w}^{\beta^{\prime}}}{\left|\vec{z}-\vec{z}_{\beta^{\prime}}\right|^{2}}\right)+\sum_{\beta^{\prime \prime}} \frac{2 P_{w}^{\beta^{\prime \prime}}}{\left|\vec{z}-\vec{z}_{\beta^{\prime \prime}}\right|^{2}}+\sum_{\beta^{\prime \prime \prime}} \frac{2 P_{w}^{\beta^{\prime \prime \prime}}}{\left|\vec{z}-\vec{z}_{\beta^{\prime \prime \prime}}\right|^{2}} .(3.6
\end{aligned}
$$


Our notation here is that $\vec{y}_{\alpha}, \vec{y}_{\alpha^{\prime}}$, etc. , denote independent sets of instanton locations for each type of index $\alpha, \alpha^{\prime}$, etc. . Likewise, the quantities $Q_{e}^{\alpha}, Q_{e}^{\alpha^{\prime}}$, etc. , denote independent sets of charges at the different sets of locations.

The equations for the 5-brane functions $H$ and $\widetilde{H}$ have Yang-Mills source terms. We shall consider the situation where the source in each equation is an $S U(2)$ Yang-Mills instanton (using a different $S U(2)$ subgroup for each equation). The use of single-charge and certain classes of multi-charge $S U(2)$ instanton solutions as sources for the 5-brane were discussed in [16, 17]. We have

$$
\begin{aligned}
H & =1+\frac{1}{4} \square_{\vec{y}} \log \left(1+\sum_{\alpha=1}^{N} \frac{\lambda_{\alpha}}{\left|\vec{y}-\vec{y}_{\alpha}\right|^{2}}\right)+\sum_{\alpha=1}^{N} \frac{1}{\left|\vec{y}-\vec{y}_{\alpha}\right|^{2}}, \\
\widetilde{H} & =1+\frac{1}{4} \square_{\vec{z}} \log \left(1+\sum_{\beta=1}^{N^{\prime}} \frac{\tilde{\lambda}_{\beta}}{\left|\vec{z}-\vec{z}_{\beta}\right|^{2}}\right)+\sum_{\beta=1}^{N^{\prime}} \frac{1}{\left|\vec{z}-\vec{z}_{\beta}\right|^{2}} .
\end{aligned}
$$

As discussed in 117, the final terms in these expressions for $H$ and $\widetilde{H}$ serve the purpose of subtracting out singular-source contributions to the functions $H$ and $\widetilde{H}$. Clearly, one can always add in any harmonic solution of the homogeneous equations $\square_{\vec{y}} H=0$ and $\square_{\vec{z}} \widetilde{H}=0$ to the solutions of the inhomogeneous equations given in (3.5). However, we are interested in the case where the sources are entirely non-singular, coming only from the Yang-Mills instantons. It turns out that the terms involving the logarithms in (3.7) actually include singular-source contributions, and the final terms in the expressions for $H$ and $\widetilde{H}$ are put in precisely to subtract these out.

Thus, as discussed in [17, the functions $H$ and $\widetilde{H}$ given in (3.7) satisfy the equations of motion throughout the space with no singularities. There are two types of phase transition that can occur in certain limits of the instanton moduli, i.e. the instanton sizes and their relative locations. If the size of an instanton shrinks to zero, the associated $H$ function becomes harmonic, with a delta-function singularity at the location of the instanton, implying that a point-like fundamental 5-brane is created [17]. The second type of phase transition occurs if two instantons coalesce, leading to the creation of a point-like fundamental 5-brane [17]. As we shall see later, the vanishing of the instanton degrees of freedom, and hence the creation of the fundamental 5-brane, turns the null area of the horizon to a non-vanishing one. 


\section{Supersymmetry}

To begin, we shall consider the situation where we take the scale sizes of the Yang-Mills instantons to zero, so that the function $H$ and $\widetilde{H}$ become harmonic, satisfying (2.3). Having studied the supersymmetry in the framework of type IIA supergravity and M-theory, and then in the heterotic framework, we shall then consider the situation when the Yang-Mills instantons replace the singular sources for the 5-branes. We shall see that the preserved supersymmetry will be the same whether or not the vanishing-instanton limit is taken.

\subsection{M-theory perspective}

When the Yang-Mills instanton scales are both set to zero, the solution in the heterotic theory can be embedded into type IIA supergravity, and hence into M-theory. We shall make use of this in order to calculate the explicit conditions for the existence of Killing spinors, by viewing the configuration as a solution in $D=11$. Then, we shall compare these explicit results with what one learns by studying the matrix of anticommutators of supercharges. This latter method can be a useful tool for determining the fraction of unbroken supersymmetry, although as we shall see, the results that come from it must be interpreted with care. Specifically, it can sometimes give a false indication of "enhanced" supersymmetry for special values of the charges, but these always turn out to be spurious, being associated with configurations with negative-mass contributions in the metric and naked singularities. (Similar issues were discussed previously in [19].)

\subsubsection{Killing spinor construction}

We may view the configuration comprising the intersection of the two 5-branes, string and a pp-wave as a solution of $D=11$ supergravity. To do this, we oxidise from $D=10$ type IIA to $D=11$ using the standard Kaluza-Klein rules:

$$
\begin{aligned}
d \hat{s}_{11}^{2} & =e^{-\frac{1}{6} \phi} d s_{10}^{2}+e^{\frac{4}{3} \phi}\left(d \xi+\mathcal{A}_{(1)}\right)^{2}, \\
\hat{F}_{(4)} & =F_{(4)}+F_{(3)} \wedge\left(d \xi+\mathcal{A}_{(1)}\right) .
\end{aligned}
$$

Thus we find that the solution in $D=11$ is given by

$$
\begin{aligned}
d \hat{s}_{11}^{2}= & K^{-\frac{2}{3}}(H \widetilde{H})^{-\frac{1}{3}}\left(-W^{-1} d t^{2}+W\left(d x+\left(W^{-1}-1\right) d t\right)^{2}\right) \\
& +K^{\frac{1}{3}} H^{\frac{2}{3}} \widetilde{H}^{-\frac{1}{3}} d \vec{y}^{2}+K^{\frac{1}{3}} H^{-\frac{1}{3}} \widetilde{H}^{\frac{2}{3}} d \vec{z}^{2}+K^{-\frac{2}{3}}(H \widetilde{H})^{\frac{2}{3}} d \xi^{2}, \\
\hat{F}_{(4)}= & \left(*_{y} d H+*_{z} d \widetilde{H}+d K^{-1} \wedge d t \wedge d x\right) \wedge d \xi
\end{aligned}
$$


where $*_{y}$ and $*_{z}$ denote the Hodge duals in the four-dimensional $y$ and $z$ subspaces, in the metrics $d y^{i} d y^{i}$ and $d z^{m} d z^{m}$ respectively. Note that here the solution describes a nonstandard intersection of two 5-branes, a membrane, and a pp-wave. We have made the specific choice of the solution where all four $\eta$ sign parameters in (2.2) are taken to be +1 . We shall discuss the effects of including the $\eta$ parameters later.

To solve for the Killing spinors, it is useful first to calculate the spin connection for the class of metrics

$$
d \hat{s}_{11}^{2}=-e^{2 A} d t^{2}+e^{2 A} W^{2}\left(d x+\left(W^{-1}-1\right) d t\right)^{2}+e^{2 B} d \vec{y}^{2}+e^{2 C} d \vec{z}^{2}+e^{2 f} d \xi^{2}
$$

taking the natural orthonormal basis $e^{0}=e^{A} d t, e^{9}=W e^{A}\left(d x+\left(W^{-1}-1\right) d t\right.$ ) (where we choose to take $x^{0}=t$ and $\left.x^{9}=x\right) ; e^{i}=e^{B} d y^{i}, e^{m}=e^{C} d z^{m}$; and $e^{\#}=e^{f} d \xi$. (There should be no confusion between vielbeins and exponentials! Note that \#, pronounced "ten," denotes the vielbein component in the extra dimension.) We find that the spin connection is

$$
\begin{aligned}
& \omega_{09}=-\frac{1}{2} e^{-B} W^{-1} \partial_{i} W e^{i}-\frac{1}{2} e^{-C} W^{-1} \partial_{m} W e^{m}, \\
& \omega_{0 i}=e^{-B}\left(-\partial_{i} A e^{0}-\frac{1}{2} W^{-1} \partial_{i} W e^{9}\right), \\
& \omega_{0 m}=e^{-C}\left(-\partial_{m} A e^{0}-\frac{1}{2} W^{-1} \partial_{m} W e^{9}\right), \\
& \omega_{9 i}=e^{-B}\left(\partial_{i} A+W^{-1} \partial_{i} W\right) e^{9}-\frac{1}{2} e^{-B} W^{-1} \partial_{i} W e^{0}, \\
& \omega_{9 m}=e^{-C}\left(\partial_{m} A+W^{-1} \partial_{m} W\right) e^{9}-\frac{1}{2} e^{-C} W^{-1} \partial_{m} W e^{0}, \\
& \omega_{i j}=e^{-B}\left(\partial_{j} B e^{i}-\partial_{i} B e^{j}\right), \quad \omega_{i m}=e^{-C} \partial_{m} B e^{i}-e^{-B} \partial_{i} C e^{m}, \\
& \omega_{m n}=e^{-C}\left(\partial_{n} C e^{m}-\partial_{m} C e^{n}\right), \quad \omega_{i \#}=-e^{-B} \partial_{i} f e^{\#}, \quad \omega_{m \#}=-e^{-C} \partial_{m} f e^{\#} .
\end{aligned}
$$

The supersymmetry transformations in $D=11$ are given by

$$
\delta \psi_{A}=D_{A} \epsilon-\frac{1}{288} \Gamma_{A}^{B C D E} \epsilon F_{B C D E}+\frac{1}{36} \Gamma^{B C D} \epsilon F_{A B C D}
$$

Consider first the $A=0$ vielbein components of this equation. Substituting the elevendimensional solution (4.2) into this, and using (4.4), we obtain

$$
\begin{aligned}
\delta \psi_{0}= & K^{\frac{1}{3}}(H \widetilde{H})^{\frac{1}{6}} \partial_{0} \epsilon \\
& -K^{-\frac{1}{6}} H^{-\frac{1}{3}} \widetilde{H}^{\frac{1}{6}}\left[\frac{1}{6} K^{-1} \partial_{i} K\left(\Gamma_{\mu i}+\epsilon_{\mu}{ }^{\nu} \Gamma_{\nu i \#}\right)\right. \\
& \left.\quad+\frac{1}{12} H^{-1} \partial_{i} H\left(\Gamma_{\mu i}+\frac{1}{6} \epsilon_{i j k \ell} \Gamma_{\mu j k \ell \#}\right)+\frac{1}{4} W^{-1} \partial_{i} W\left(\Gamma_{0 i}+\Gamma_{9 i}\right)\right] \epsilon \\
& -K^{-\frac{1}{6}} H^{\frac{1}{6}} \widetilde{H}^{-\frac{1}{3}}\left[\frac{1}{6} K^{-1} \partial_{m} K\left(\Gamma_{\mu m}+\epsilon_{\mu}{ }^{\nu} \Gamma_{\nu m \#}\right)\right. \\
& \left.+\frac{1}{12} \widetilde{H}^{-1} \partial_{m} \widetilde{H}\left(\Gamma_{\mu m}+\frac{1}{6} \epsilon_{m n p q} \Gamma_{\mu n p q \#}\right)+\frac{1}{4} W^{-1} \partial_{m} W\left(\Gamma_{0 m}+\Gamma_{9 m}\right)\right] \epsilon .
\end{aligned}
$$


From this, we see that we shall have solutions of $\delta \psi_{0}=0$ if $\partial_{0} \epsilon=0$, and the following conditions hold:

$$
\begin{array}{lc}
\left(\Gamma_{i}+\frac{1}{6} \epsilon_{i j k \ell} \Gamma_{j k \ell \#}\right) \epsilon=0, & \left(\Gamma_{m}+\frac{1}{6} \epsilon_{m n p q} \Gamma_{n p q \#}\right) \epsilon=0 \\
\left(\Gamma_{0}+\Gamma_{9 \#}\right) \epsilon=0, & \left(\Gamma_{0}+\Gamma_{9}\right) \epsilon=0 .
\end{array}
$$

Note that the first two conditions come respectively from the coefficients of $\partial_{i} H$ and $\partial_{m} \widetilde{H}$ in (4.6), while the last two conditions come respectively from the coefficients of $\left(\partial_{i} K, \partial_{m} K\right)$ and $\left(\partial_{i} W, \partial_{m} W\right)$. Thus if any of the functions $H, \widetilde{H}, K$ or $W$ is trivial (i.e. equal to 1 ), then the associated condition in (4.7) will be absent. Note also that in deriving separate conditions associated with each function, we have implicitly assumed that the functions are independent (i.e. not proportional to one another).

Proceeding with the $A=9, i, m$ and $\xi$ components of $\delta \psi_{A}$ in a similar fashion, we find that the conditions for the existence of Killing spinors are just precisely those already found in (4.7), together with

$$
\epsilon=K^{-\frac{1}{6}}(H \tilde{H})^{-\frac{1}{12}} W^{-\frac{1}{4}} \epsilon_{0},
$$

where $\epsilon_{0}$ is a constant spinor.

If we take the indices $i$ and $m$ in the $\vec{y}$ and $\vec{z}$ spaces to range over the values $(1,2,3,4)$ and $(5,6,7,8)$ respectively, it follows from (4.7) that the conditions for the existence of Killing spinors are that the constant spinor $\epsilon_{0}$ must satisfy

$$
\begin{aligned}
H: & \epsilon_{0}=-\Gamma_{1234 \#} \epsilon_{0}, \\
\widetilde{H}: & \epsilon_{0}=-\Gamma_{5678 \#} \epsilon_{0}, \\
K: & \epsilon_{0}=\Gamma_{09 \#} \epsilon_{0}, \\
W: & \epsilon_{0}=\Gamma_{09} \epsilon_{0},
\end{aligned}
$$

where we have made explicit which condition is associated with which metric function.

Any one of the four equations in (4.9) by itself has 16 independent solutions for $\epsilon_{0}$. Thus with just one of the four charges turned on, the solution preserves $\frac{1}{2}$ of the elevendimensional supersymmetry. The number of solutions that one gets when more charges are turned on depends on various factors, including one's choice of gamma-matrix conventions. In particular, one should bear in mind that the product $\Gamma_{0123456789 \#}$ must be either $+\mathbb{1}$ or $-\mathbb{1}$. If we make the convention choice

$$
\Gamma_{0123456789 \#}=+\mathbb{1}
$$

then we find, for example, that the conditions from $H$ and from $\widetilde{H}$ are equivalent, and so introducing the charge for $\widetilde{H}$ as well as for $H$ would yield no further constraints. On the 
other hand, if the opposite gamma-matrix convention to (4.10) were chosen, then intro-

ducing $\widetilde{H}$ as well as $H$ would cause all Killing spinors to be lost. We shall not enumerate here all the supersymmetry fractions for the various possible non-vanishing sets of charges, since the results can be summarised more succinctly later. Let us just remark for now that with optimally-chosen conventions, one finds that the solution with all four charges active preserves $\frac{1}{8}$ of the supersymmetry.

We observed in section 2 that there are actually 16 independent solutions to the bosonic equations of motion that follow from (2.1), where we allow the independent choice of +1 or -1 for each of the parameters $\eta, \tilde{\eta}, \eta_{e}$ and $\eta_{w}$ in (2.2). Thus our specific choice in (4.2) corresponds to $\left(\eta, \tilde{\eta}, \eta_{e}, \eta_{w}\right)=(+1,+1,+1,+1)$. It is clear that when we reinstate the parameters, the conditions (4.9) will be replaced by

$$
\begin{aligned}
H: & \epsilon_{0}=-\eta \Gamma_{1234 \#} \epsilon_{0}, \\
\widetilde{H}: & \epsilon_{0}=-\tilde{\eta} \Gamma_{5678 \#} \epsilon_{0}, \\
K: & \epsilon_{0}=\eta_{e} \Gamma_{09 \#} \epsilon_{0}, \\
W: & \epsilon_{0}=\eta_{w} \Gamma_{09} \epsilon_{0} .
\end{aligned}
$$

We shall return to a discussion of the possible sign choices later.

Note that using (4.10), we can replace the gamma-matrix combinations $\Gamma_{1234 \#}$ and $\Gamma_{5678 \#}$ in (4.11) by $\Gamma_{056789}$ and $\Gamma_{012349}$ respectively. This then means that the indices on the first three gamma-matrix combinations in (4.11) can be viewed as lying in the worldvolumes of the two 5-branes and the membrane respectively. The last combination, for $W$, lies in the plane in which the pp-wave propagates.

\subsubsection{Superalgebra analysis}

It is now instructive to compare the explicit results that we have obtained for the Killing spinors with what one learns from the eleven-dimensional supersymmetry algebra. We shall follow some of the notation and conventions of [20]. One finds that the anticommutator of the supercharges $Q$ gives the expression

$$
\{Q, Q\}=C\left(\Gamma^{M} P_{M}+\frac{1}{2 !} \Gamma^{M_{1} M_{2}} Z_{M_{1} M_{2}}+\frac{1}{5 !} \Gamma^{M_{1} \cdots M_{5}} Z_{M_{1} \cdots M_{5}}\right)
$$

where $C$ is the charge-conjugation matrix, which can be taken to be $C=\Gamma^{0}$, and $Z_{M_{1} M_{2}}$ and $Z_{M_{1} \cdots M_{5}}$ are 2-form and 5-form charges. In the present case, where we have two 5-branes and a membrane supported by $F_{(4)}$ in $D=11$, the charges will be given by the asymptotic integrals of the three terms in $F_{(4)}$ given in (4.2). Let us call the 5-brane and membrane 
charges $q_{5}, \tilde{q}_{5}$ and $q_{2}$ respectively. Thus we will have non-zero $Z$ 's given by

$$
Z_{12349}=q_{5}, \quad Z_{56789}=\tilde{q}_{5}, \quad Z_{9 \#}=q_{2}
$$

In addition, the pp-wave will contribute to the momentum $P_{M}$ in its direction of propagation, and so

$$
P_{9}=q_{w}
$$

We may choose a basis for the eleven-dimensional gamma matrices where

$$
\begin{aligned}
\Gamma_{012349} & =\operatorname{diag}(1,1,1,1,-1,-1,-1,-1) \otimes \mathbb{1}_{4}, \\
\Gamma_{056789} & =\operatorname{diag}(1,1,-1,-1,1,1,-1,-1) \otimes \mathbb{1}_{4}, \\
\Gamma_{09 \#} & =\operatorname{diag}(1,1,-1,-1,-1,-1,1,1) \otimes \mathbb{1}_{4}, \\
\Gamma_{09} & =\operatorname{diag}(1,-1,-1,1,-1,1,1,-1) \otimes \mathbb{1}_{4},
\end{aligned}
$$

since $\Gamma_{012349}, \Gamma_{056789}, \Gamma_{09 \#}$ and $\Gamma_{09}$ all commute with one another. We therefore find that

$$
\begin{gathered}
\{Q, Q\}=\operatorname{diag}\left(E-q_{5}-\tilde{q}_{5}-q_{2}-q_{w}, E-q_{5}-\tilde{q}_{5}-q_{2}+q_{w}, E-q_{5}+\tilde{q}_{5}+q_{2}+q_{w},\right. \\
E-q_{5}+\tilde{q}_{5}+q_{2}-q_{w}, E+q_{5}-\tilde{q}_{5}+q_{2}+q_{w}, E+q_{5}-\tilde{q}_{5}+q_{2}-q_{w}, \\
\left.E+q_{5}+\tilde{q}_{5}-q_{2}-q_{w}, E+q_{5}+\tilde{q}_{5}-q_{2}-q_{w}\right) \otimes \mathbb{1}_{4} .
\end{gathered}
$$

where the total energy $E$ is given by

$$
E=p_{5}+\tilde{p}_{5}+p_{2}+p_{w}
$$

The quantities $p_{5}, \tilde{p}_{5}, p_{2}$ and $p_{w}$ are the individual contributions to the ADM mass coming from the two 5-branes, the membrane and the pp-wave. They correspond directly to the overall asymptotic coefficients of leading-order inverse power-law coordinate dependences of the four metric functions $H, \widetilde{H}, K$ and $W$. The corresponding charges $q_{5}, \tilde{q}_{5}, q_{2}$ and $q_{w}$ are related to them by

$$
\left(q_{5}, \tilde{q}_{5}, q_{2}, q_{w}\right)=\left(\eta p_{5}, \tilde{\eta} \tilde{p}_{5}, \eta_{e} p_{2}, \eta_{w} p_{w}\right)
$$

where $\left(\eta, \tilde{\eta}, \eta_{e}, \eta_{w}\right)$ are the $16 \pm$ sign choices in the bosonic solutions that we discussed previously.

Consider first the case where $\left(\eta, \tilde{\eta}, \eta_{e}, \eta_{w}\right)=(+1,+1,+1,+1)$. We then find that the anticommutator 4.16) is given by

$\{Q, Q\}=2 \operatorname{diag}\left(0, p_{w}, \tilde{p}_{5}+p_{2}+p_{w}, \tilde{p}_{5}+p_{2}, p_{5}+p_{2}+p_{w}, p_{5}+p_{2}, p_{5}+\tilde{p}_{5}, p_{5}+\tilde{p}_{5}+p_{w}\right) \otimes \mathbb{1}_{4}$. 
Clearly in general, namely with all four parameters non-zero, this will have just 4 zero eigenvalues, giving a counting of $\frac{1}{8}$ unbroken supersymmetry that is in precise agreement with our findings from the explicit solutions for the Killing spinors. For the various possible combinations of non-vanishing subsets of parameters $\left(p_{5}, \tilde{p}_{5}, p_{2}, p_{w}\right)$, the corresponding numbers of zero eigenvalues in (4.19) can be read off. In each case it is easy to verify that the counting agrees precisely with our previous derivation of the constraint equations (4.9) for the Killing spinors, provided that, as usual, the spurious zero eigenvalues that could apparently be achieved by allowing negative $p$ parameters are discarded.

For a total of 8 of the 16 possible sign choices in (4.18), the story is similar. It is best summarised by discussing the eigenvalues of $\{Q, Q\}$, rather than keeping track of the ordering of diagonal entries, which are permuted around in the various cases. Thus we may say that for 8 of the sign choices, we obtain the eigenvalues

$$
\lambda=2\left(0, p_{5}+p_{2}, \tilde{p}_{5}+p_{2}, p_{5}+\tilde{p}_{5}, p_{w}, p_{5}+p_{2}+p_{w}, \tilde{p}_{5}+p_{2}+p_{w}, p_{5}+\tilde{p}_{5}+p_{w}\right),
$$

each occurring with degeneracy 4. (This is the same as the set of eigenvalues in the specific example (4.19).) For these supersymmetric solutions, the mass/charge relations are given by

$$
E \equiv p_{5}+\tilde{p}_{5}+p_{2}+p_{w}=q_{2} \pm\left(q_{5}+\tilde{q}_{5}\right) \pm q_{w}, \quad \text { or } \quad-q_{2} \pm\left(q_{5}-\tilde{q}_{5}\right) \pm q_{w} .
$$

For the remaining 8 possibilities, the eigenvalues are given by

$$
2\left(p_{2}, p_{5}, \tilde{p}_{5}, p_{5}+\tilde{p}_{5}+p_{2}, p_{2}+p_{w}, p_{5}+p_{w}, \tilde{p}_{5}+p_{w}, p_{5}+\tilde{p}_{5}+p_{2}+p_{w}\right)
$$

and hence these solutions break all the supersymmetry if all the charges are non-vanishing. For these non-supersymmetric solutions the mass/charge relations are given by

$$
E \equiv p_{5}+\tilde{p}_{5}+p_{2}+p_{w}=q_{2} \pm\left(q_{5}-\tilde{q}_{5}\right) \pm q_{w}, \quad \text { or } \quad-q_{2} \pm\left(q_{5}+\tilde{q}_{5}\right) \pm q_{w} .
$$

Naively, it might seem that we could achieve different, sometimes unusual, fractions of unbroken supersymmetry by making certain special non-vanishing choices for the $\left(p_{5}, \tilde{p}_{5}, p_{2}, p_{w}\right)$ parameters. For example, choosing $p_{5}=\tilde{p}_{5}=-p_{2}$, with $p_{w}=0$, in (4.20), we could apparently get 24 zero eigenvalues and hence $\frac{3}{4}$ preserved supersymmetry. On the other hand, we saw no indication at all from the explicit solutions for the Killing spinors that such "supersymmetry enhancements" could occur at special values of the charges.[

\footnotetext{
${ }^{3}$ We did remark at the stage when we obtained the conditions (4.7) for the existence of Killing spinors that we were assuming that the functions $H, \widetilde{H}, K$ and $W$ were not proportional to one another. It is crucial to appreciate that the quantities $p_{5}, \tilde{p}_{5}, p_{2}$ and $p_{w}$ are precisely the coefficients appearing in these functions, and so there can be no possibility of two of the functions being proportional in the kinds of cases we are considering here, where one of their $p$ coefficients is the negative of the other.
} 
The resolution to this puzzle, as discussed in [19, 21], is that the occurrence of zero eigenvalues in the anticommutator of supercharges can sometimes give a false impression of the existence of additional Killing spinors, and here we have encountered precisely such an example. The reason why the supercharge argument is unreliable here is that in order to achieve the extra zeroes in (4.19) it was necessary to have at least one of the contributions $\left(p_{5}, \tilde{p}_{5}, p_{2}, p_{w}\right)$ that appear in the energy (4.17) be negative. This means that the associated harmonic function will have a negative asymptotic coefficient, and hence it means that there will be a naked singularity in the metric in some region. Under such circumstances, the assumed conditions under which one derives a relation between zeroes of the anticommutator (4.12) and unbroken supersymmetry generators are violated, and so one cannot trust the result. The fact that no additional Killing spinors actually arise, as seen from our earlier explicit calculation, shows that this is indeed what has happened in this case.

\subsection{Heterotic theory perspective}

To study the fractions of preserved supersymmetry in the heterotic theory, we can use the same Killing-spinor calculations as we did before, but now we impose the additional ten-dimensional chirality condition on the Killing spinor,

$$
\epsilon_{0}=\Gamma_{\#} \epsilon_{0}
$$

(Of course we might instead impose this condition with a minus sign, depending on our conventions.) Thus the number of independent components of unbroken supersymmetry is determined by solving the equations (4.9), together with (4.24). As usual, if any of the charges in (4.9) is zero, then the associated condition is omitted. Rather than stating the results for the various supersymmetry fractions here, it is more convenient first to give the discussion of the anticommutator of supercharges in this heterotic case. As in the previous M-theory discussion, we again find that the two approaches agree, provided that we discard any apparent supersymmetry enhancements that would naively appear to occur in the $\{Q, Q\}$ eigenvalue calculation when there are negative energy contributions.

Since we are not for now concerned with the contributions of the Yang-Mills fields in the anticommutator algebra $\{Q, Q\}$, we can make use of the same formalism (4.12) as in $D=11$, but with the additional requirement that we should project all matrices onto the positive eigenspace of the chirality operator $\Gamma_{\#}$. From (4.15), we see that in the gamma matrix conventions we are using here $\Gamma_{\#}$ will be given by

$$
\Gamma_{\#}=\operatorname{diag}(1,-1,1,-1,1,-1,1,-1) \otimes \mathbb{1}_{4} .
$$


The projection onto the positive eigenspace of $\Gamma_{\#}$ therefore amounts to keeping only the 1'st, 3'rd, 5'th and 7'th entries in (4.16). It turns out that for 4 out of the possible 16 sign choices in $\left(q_{5}, \tilde{q}_{5}, q_{2}, q_{w}\right)=\left( \pm p_{5}, \pm \tilde{p}_{5}, \pm p_{2}, \pm p_{w}\right)$ we obtain eigenvalues

$$
\lambda=2\left(0, \tilde{p}_{5}+p_{2}, p_{5}+p_{2}+p_{w}, p_{5}+\tilde{p}_{5}\right)
$$

for $\{Q, Q\}$, each with degeneracy 4. This expression summarises all the information about the possible supersymmetry fractions that can be achieved; as usual, all the quantities $\left(p_{5}, \tilde{p}_{5}, p_{2}, p_{w}\right)$ should be considered to be non-negative. The various supersymmetry fractions implied by taking all possible non-zero subsets of the $\left(p_{5}, \tilde{p}_{5}, p_{2}, p_{w}\right)$ agree completely with those obtained by explicitly solving the Killing-spinor equations. The mass/charge relation for these supersymmetric solutions is given by

$$
E \equiv p_{5}+\tilde{p}_{5}+p_{2}+p_{w}=\left(q_{2}+q_{w}\right) \pm\left(q_{5}+\tilde{q}_{5}\right), \quad \text { or } \quad-\left(q_{2}+q_{w}\right) \pm\left(q_{5}-\tilde{q}_{5}\right)
$$

Note that the other 12 possible sign choices in $\left(q_{5}, \tilde{q}_{5}, q_{2}, q_{w}\right)=\left( \pm p_{5}, \pm \tilde{p}_{5}, \pm q_{2}, \pm q_{w}\right)$ divide into three further sets of 4 , with each set leading to eigenvalues as follows:

$$
\begin{aligned}
\lambda & =\left(p_{5}+p_{2}, \tilde{p}_{5}+p_{2}, p_{w}, p_{5}+\tilde{p}_{5}+p_{w}\right), \\
\lambda & =\left(p_{2}, p_{5}+p_{w}, \tilde{p}_{5}+p_{w}, p_{5}+\tilde{p}_{5}+p_{2}\right), \\
\lambda & =\left(p_{5}, \tilde{p}_{5}, p_{2}+p_{w}, p_{5}+\tilde{p}_{5}+p_{2}+p_{w}\right) .
\end{aligned}
$$

Thus with all four charges turned on, none of these other sets gives rise to any preserved supersymmetry. Note also that if we make the opposite sign choice for the chirality projection, then the same sets of eigenvalues (4.26) and (4.28) occur, but now with different combinations of sign choice in $\left(q_{5}, \tilde{q}_{5}, q_{2}, q_{w}\right)=\left( \pm p_{5}, \pm \tilde{p}_{5}, \pm q_{2}, \pm q_{w}\right)$ being associated with each set of eigenvalues.

The above example provides another manifestation of a supersymmetry rule obtained in [11. Namely, when a new intersecting ingredient is introduced in a set of intersections, if it breaks a further half of the supersymmetry then the structure of the eigenvalues of $\{Q, Q\}$ is independent of the sign of the new charge. If, on other hand, the introduction of the new charge does not break supersymmetry further, then it would break the supersymmetry completely if it were instead introduced with the opposite sign. Thus for $N$ intersecting objects that preserve $1 / 2^{n}$ of the supersymmetry with $n \leq N, 2^{n}$ out of the $2^{N}$ possible choices of solutions are supersymmetric while the rest are non-supersymmetric.

Finally, in our discussion of supersymmetry in the heterotic framework, we examine the situation where we include the Yang-Mills fields, and consider the solutions where $H$ 
and $\widetilde{H}$ are non-singular functions with Yang-Mills instanton sources, as given in (3.7). The supersymmetry transformation rule for the gravitino is unchanged from the one in the $N=1$ truncation of the type II theory, and so our previous calculation of the supersymmetry in the gravitino sector goes through unchanged. (In the derivation of the Killing spinors, for example in (4.6), it was not important that the functions $H$ and $\widetilde{H}$ be harmonic.) However, we now have to consider also the supersymmetry transformations of the gaugini $\chi^{a}$ and $\chi^{\alpha}$, which are the superpartners of the $S U(2)$ Yang-Mills fields $B_{(1)}^{a}$ and $\mathcal{B}_{(1)}^{\alpha}$ respectively. These take the form

$$
\delta \chi^{a}=G_{i j}^{a} \Gamma_{i j} \epsilon, \quad \delta \chi^{\alpha}=\mathcal{G}_{m n}^{\alpha} \Gamma_{m n} \epsilon
$$

Now, if $G_{i j}^{a}$ is self-dual or anti-self-dual, we shall therefore find that the Killing spinors $\epsilon$ should satisfy

$$
\left(\Gamma_{i j} \pm \frac{1}{2} \epsilon_{i j k \ell} \Gamma_{k \ell}\right) \epsilon=0
$$

respectively, with similar conclusions in the $\vec{z}$ space for $\delta \chi^{\alpha}$. Equivalently, we can express these conditions as

$$
\epsilon=\mp \Gamma_{1234} \epsilon, \quad \epsilon=\mp \Gamma_{5678} \epsilon .
$$

Bearing in mind that we also have the chirality condition (4.24) (or its opposite), we see that the conditions (4.31) are of the same form as the ones already encountered in (4.11). Thus the question of whether the solutions are supersymmetric or not comes down to the issue of achieving a proper correlation of signs, with the self-duality or anti-self-duality choice for the Yang-Mills instantons being correlated with the chirality sign convention for the spinors of the heterotic theory. Provided the signs are properly chosen, the preserved supersymmetry fractions for the Yang-Mills instanton-supported solutions in the heterotic theory will be the same as for their corresponding singular-source limits.

\subsection{Further comments}

In the previous discussion we demonstrated that the correspondence between the counting of zero-eigenvalues in the anticommutator $\{Q, Q\}$ and the counting of Killing spinors holds if the energy contribution from each intersecting ingredient is non-negative. One cannot trust any additional zero-eigenvalues that arise by virtue of having any negative-energy contributions from any of the intersecting ingredients. It should be emphasised, however, that one does not always get the wrong conclusion from the $\{Q, Q\}$ calculation when there are negative-energy contributions. For example, for a simple extremal $p$-brane solution preserving $\frac{1}{2}$ supersymmetry the analogous result is $\{Q, Q\}=2(0, p) \times \mathbb{1}_{16}$, and this correctly implies that there will continue to be 16 unbroken components of supersymmetry even if the 
mass is taken to be negative. (The Killing spinor equation continues to admit 16 solutions, even if one sets the mass negative.) But the $\{Q, Q\}$ calculation is giving the "correct conclusion for the wrong reason" if the mass is negative. On the other hand, it seems that in all cases where there appear to be enhanced supersymmetry fractions for particular tuned sets of charges that involve negative-energy contributions, the conclusion is always wrong.

Although Killing spinor solutions still exist even if an intersecting ingredient contributes a negative energy, the behavior of the Killing spinor will become singular. To see this, recall that the Killing spinor solution (4.8) holds regardless of the detailed structure of the harmonic functions $K, H, \widetilde{H}$ or $W$. When each intersecting ingredient has positive energy, these harmonic functions take values between 1 and $\infty$, and hence the Killing spinors are finite. On the other hand, if any intersecting object contributes a negative energy, then the associated harmonic function takes values between 1 and 0 , with a naked singularity occurring at the latter value. As can be seen from (4.8), the Killing spinor blows up at the naked singularity.

In most examples, requiring the regularity of the Killing spinor leads to a positive energy contribution, which in turn implies cosmic censorship [24]. However, as observed in [21, when a 5-brane with negative mass is supported by a Yang-Mills instanton of sufficient scale size, the previous singularity in metric can be smeared out by the instanton, and hence the Killing spinor is also well-behaved. In this case, the continued violation of the relation between the zero-eigenvalue counting in $\{Q, Q\}$ and the counting of Killing spinors is caused by the fact that the energy-momentum tensor of the Yang-Mills instanton fields now violates the positive-energy condition [21].

For $N$ intersecting objects that preserve $1 / 2^{N}$ of the supersymmetry, the eigenvalues of the anticommutator $\{Q$,$\} have the form$

$$
\lambda=\left(E \pm p_{1} \pm \cdots \pm p_{N}\right)=2\left(0, p_{1}, p_{2}, \ldots, p_{N}, p_{1}+p_{2}, p_{1}+p_{3}, \ldots, p_{1}+\cdots p_{N}\right)
$$

where the total energy is $E=\sum_{i} p_{i}$. Thus the anticommutator $\{Q, Q\}$ would have negative eigenvalues if any of the objects contributes a negative energy. On the other hand, if the $N$ intersecting objects preserve $1 / 2^{n}$ of the supersymmetry with $n<N$, then $\{Q, Q\}$ can still remain positive even if some of the individual energy contributions are negative, since in these cases, not all the individual energy contributions appear in isolation as eigenvalues. For example, the eigenvalues in (4.19) will remain non-negative even if we set $q_{2}+q_{5}=$ 0 . The phenomenon was observed in [25] in the context of 4-charge black holes in fourdimensional heterotic string theory, where the eigenvalues of $\{Q, Q\}$ are $2\left\{0, p_{1}+p_{2}, p_{3}+\right.$ $\left.p_{4}, p_{1}+p_{2}+p_{3}+p_{4}\right\}$ and the energy is $E=p_{1}+p_{2}+p_{3}+p_{4}$. Thus one can obtain 
a massless black hole by setting $p_{1}+p_{2}=0$ and $p_{3}+p_{4}=0$, without there being any negative eigenvalues. Another example in the literature is the dyonic string in $N=1$ supergravity, for which $\{Q, Q\}$ has eigenvalues $2\left\{0, p_{1}+p_{2}\right\}$, with energy $E=p_{1}+p_{2}$ [16, 21]. It follows that the dyonic string becomes tensionless when $p_{1}+p_{2}=0$. (Of course as usual, one should not take the occurrence of extra zero-eigenvalues in these limits as indicating enhanced supersymmetry.) It seems that solutions such as the above massless ones, where no negative eigenvalues occur in $\{Q, Q\}$, may have a more solid relation to states in the quantum theory than ones where masslessness is achieved at the price of negative eigenvalues.

In our present case in this paper, from an M-theory perspective, there is no choice of parameters such that the energy $E=p_{5}+\widetilde{p}_{5}+p_{2}+p_{w}$ vanishes while the eigenvalues in the associated anticommutator (4.19) all remain positive. On the other hand, from the heterotic perspective the eigenvalues in the superalgebra are truncated to (4.26), and hence it is possible to obtain a massless solution with purely non-negative eigenvalues in the associated superalgebra. Of course achieving the massless solution requires negative-energy contributions from some of the intersecting ingredients, and hence either the Killing spinor blows up, or the Yang-Mills energy density becomes negative. Such a pathology may imply a phase transition of the type discussed in 28, 16.

\section{Near-horizon structure}

For a single-center configuration, the solutions to equations (3.5) can be taken to be:

$$
\begin{aligned}
& K=\left(1+\frac{2 Q_{e}}{r^{2}}\right)\left(1+\frac{2 Q_{e}^{\prime}}{\rho^{2}}\right), \quad W=\left(1+\frac{2 Q_{w}}{r^{2}}\right)\left(1+\frac{2 Q_{w}^{\prime}}{\rho^{2}}\right), \\
& H=1+\frac{2\left(r^{2}+2 a^{2}\right)}{\left(r^{2}+a^{2}\right)^{2}}, \quad \widetilde{H}=1+\frac{2\left(\rho^{2}+2 b^{2}\right)}{\left(\rho^{2}+b^{2}\right)^{2}},
\end{aligned}
$$

where $r^{2}=y^{i} y^{i}$ and $\rho^{2}=z^{m} z^{m}$. Here $a$ and $b$ are the sizes of the instantons supporting each of the 5 -branes.

Let us now consider what happens if the two instanton sizes $a$ and $b$ vanish. In this situation, the functions $H$ and $\widetilde{H}$ become harmonic functions. Let us consider the horizon region with $r \rho \rightarrow 0$ and $r / \rho$ non-vanishingly finite. In this region, the additive constants " 1 " in these two functions $H$ and $\widetilde{H}$ can be dropped. Also $K \sim 4 Q_{e} Q_{e}^{\prime} /\left(r^{2} \rho^{2}\right)$. In this region the dilaton scalar $\phi$ becomes constant, and the metric becomes (for simplicity, we consider $\left.Q_{e} Q_{e}^{\prime}=1\right)$

$$
d s_{10}^{2}=d s_{4}^{2}+2 d \Omega_{3}^{2}+2 d \widetilde{\Omega}_{3}^{2}
$$


where $d \Omega_{3}^{2}$ and $d \widetilde{\Omega}_{3}^{2}$ are the metrics for unit 3-spheres, and

$$
d s_{4}^{2}=\frac{1}{4} r^{2} \rho^{2}\left(-W^{-1} d t^{2}+W\left(d x+\left(W^{-1}-1\right) d t\right)^{2}\right)+\frac{2 d r^{2}}{r^{2}}+\frac{2 d \rho^{2}}{\rho^{2}} .
$$

The four-dimensional configuration (5.3) is the solution to the Lagrangian

$$
e^{-1} \mathcal{L}=R-\frac{1}{2}(\partial \phi)^{2}-\frac{1}{2} e^{2 \phi}(\partial \chi)^{2}+m^{2} e^{\phi}
$$

which is the scalar Lagrangian of four-dimensional $S U(2) \times S U(2)$ gauged supergravity, constructed in [29].

Making the coordinate transformation $y=\log (r \rho)$ and $z=\log (r / \rho)$, the metric (5.3) becomes

$$
\begin{aligned}
& d s_{4}^{2}=\frac{1}{4} e^{2 x}\left(-W^{-1} d t^{2}+W\left(d x+\left(W^{-1}-1\right) d t\right)^{2}\right)+d y^{2}+d z^{2}, \\
& W=\left(1+Q_{w} e^{-y-z}\right)\left(1+Q_{w}^{\prime} e^{-y+z}\right) .
\end{aligned}
$$

When $Q_{w}=Q_{w}^{\prime}=0$, the four-dimensional metric becomes $\mathrm{AdS}_{3} \times S^{1}$, as discussed in [22]. (Various intersections whose near horizon structures give rise to an $\mathrm{AdS}_{3}$ spacetime were given in [9].) When the 1's are dropped from $W$, the above spacetime becomes $\mathrm{K}_{3} \times S^{1}$, where $\mathrm{K}_{D}$ denotes the generalised Kaigorodov metric in $D$ dimensions [30]. Note that $\mathrm{K}_{3}$ is locally equivalent to the BTZ black hole constructed in [31]. The near-horizon structure of this intersection was also discussed in [23], from an eleben-dimensional point of view.

The area of the horizon for the metric (5.2), and hence the entropy, is proportional to $\sqrt{Q_{w} Q_{w}^{\prime}}$. On the other hand, when the instanton sizes $a$ and $b$ are non-vanishing, the area of the horizon, and hence the corresponding entropy, would be zero. An analogous phase transition occurs also when two instantons coalesce, which increases the area of the horizon. The entropy associated with the non-vanishing area of the horizon can be understood from the two-dimensional boundary conformal field theory of the $\mathrm{AdS}_{3}$ spacetime.

Note that the Lagrangian (3.1) also admits a different type of four-object intersection, namely a string, 5-brane, pp-wave and NUT. This intersection is of the standard type, and gives rise to a 4-charge black hole [26] in $D=4$. The near-horizon structure is $\mathrm{BTZ} \times\left(S^{3} / Z_{n}\right) \times E_{4}$ [27]. By contrast, the near-horizon structure of the four-object intersection discussed in this paper is $\mathrm{K}_{3} \times S^{3} \times S^{3} \times S^{1}$.

\section{Conclusions}

In this paper, we have constructed extremal solutions in $D=10$, comprising the nonstandard intersection of two 5-branes together with a string and a pp-wave. This can arise 
as a solution in the $N=1$ truncation of the type II theory, with singular sources for all the ingredients in the intersection. This configuration can be oxidised to a solution in $D=11$, where the string now becomes a membrane living in the common world-volume directions of the 5-branes. It can instead be viewed as a solution in the heterotic theory, in which case it is possible to replace the singular sources for the 5-branes by self-dual Yang-Mills instantons. An unusual feature here is that, owing to the non-standard nature of the 5-brane intersection, in which they have non-overlapping 4-dimensional transverse spaces, there can be a separate $S U(2)$ instanton for each 5-brane. This solution therefore makes use of an $S U(2) \times S U(2)$ subgroup of the $E_{8} \times E_{8}$ or $S O(32)$ gauge group of the heterotic string.

We presented a detailed discussion of the supersymmetry of the intersecting solution. In particular, we compared the results from an explicit construction of the Killing spinors with a counting of the zero eigenvalues of the anticommutator of supercharges, $\{Q, Q\}$. We showed that the two are in agreement, provided one discounts as "spurious" the additional zero eigenvalues of $\{Q, Q\}$ that can arise for special "tuned" non-vanishing values for certain of the charges. We argued that, as discussed in previous examples in the literature [19, 21], the naive counting of zero eigenvalues of $\{Q, Q\}$ can give misleading results, if any of the components in the intersection is giving a negative contribution to the total energy. Having exhibited this phenomenon in specific examples, the implication is that one should always treat apparent supersymmetry enhancements seen from supercharge anticommutators with suspicion, unless there is some compelling argument for why they are not spurious.

\section{Acknowledgments}

We are grateful to Mirjam Cvetič for valuable discussions on Killing spinors and the Bogomoln'yi bound in four-dimensional black-hole solutions, and to Joachim Rahmfeld for raising again a question about supersymmetry enhancements.

\section{References}

[1] A. Dabholkar, G.W. Gibbons, J.A. Harvey and F. Ruiz Ruiz, Superstrings and solitons, Nucl. Phys. B340 (1990) 33.

[2] A. Strominger, Heterotic solitons, Nucl. Phys. B343 (1990) 167, Erratum-ibid. B353 (1991) 565 . 
[3] M.J. Duff and K.S. Stelle, Multi-membrane solutions of $D=11$ supergravity, Phys. Lett. B253 (1991) 113.

[4] R. Güven, Black p-brane solitons of $D=11$ supergravity theory, Phys. Lett. B276 (1992) 49 .

[5] A.A. Tseytlin, Harmonic superpositions of M-branes, Nucl. Phys. B475 (1996) 149, hep-th/9604035.

[6] M.J. Duff, R.R. Khuri and J.X. Lu String solitons, Phys. Rep. 259 (1995) 213, hepth/9412184.

[7] M. Cvetič and D. Youm, All the static spherically symmetric black holes of heterotic string on a six torus, Nucl. Phys. B472 (1996) 249, hep-th/9512127.

[8] E. Bergshoeff, M. de Roo, E. Eyras, B. Janssen, J.P. van der Schaar, Multiple intersections of D-branes and M-branes, Nucl. Phys. B494 (1997) 119, hep-th/9612095.

[9] H.J. Boonstra, B. Peeters and K. Skenderis, Brane intersections, anti-de Sitter spacetimes and dual superconformal theories, Nucl. Phys. B533 (1998) 127, hep-th/9803231.

[10] H. Lü and C.N. Pope, p-brane solitons in maximal supergravities, Nucl. Phys. B465 (1996) 127, hep-th/9512012.

[11] H. Lü, C.N. Pope, T.A Tran and K.W. Xu, Classification of p-branes, NUTs, waves and intersections, Nucl. Phys. B511 (1998) 98, hep-th/9708055.

[12] R.R Khuri, A comment on string solitons, Phys. Rev. D48 (1993) 2947, hepth/9305143.

[13] K. Behrndt, E. Bergshoeff and B. Janssen, Intersecting D-branes in ten and six dimensions, Phys. Rev. D55 (1997) 3785, hep-th/9604168.

[14] J.P. Gauntlett, D.A. Kastor and J. Traschen, Overlapping branes in M-theory, Nucl. Phys. B478 (1996) 544, hep-th/9604179.

[15] M.J. Duff, P. Hoxha, H. Lü, R.R. Martinez-Acosta, C.N. Pope, A lattice universe from M-theory, Phys. Lett. B451 (1999) 38, astro-ph/9712301.

[16] M.J. Duff, H. Lü and C.N. Pope, Heterotic phase transitions and singularities of the gauge dyonic string, Phys. Lett. B378 (1996) 101, hep-th/9603037. 
[17] E. Lima, H. Lü, B.A. Ovrut and C.N. Pope, Instanton moduli and brane creation, to appear in Nucl. Phys. B, hep-th/9903001.

[18] H. Lü and C.N. Pope, Multi-scalar p-brane solitons, Int. J. Mod. Phys. A12 (1997) 437, hep-th/9512153.

[19] H. Lü and C.N. Pope, p-brane taxonomy, in "Trieste 1996, High energy physics and cosmology," hep-th/9702086.

[20] J.P. Gauntlett and C.M. Hull, BPS states with extra supersymmetry, hep-th/9909098.

[21] M.J. Duff, James T. Liu, H. Lü, C.N. Pope, Gauged dyonic strings and their global limit, Nucl. Phys. B529 (1998) 137, hep-th/9711089.

[22] P.M. Cowdall, P.K. Townsend, Gauged supergravity vacua from intersecting branes, Phys. Lett. B429 (1998) 281, Erratum-ibid. B434 (1998) 458, hep-th/9801165.

[23] J.P. Gauntlett, R.C. Myers and P.K. Townsend, Supersymmetry of rotating branes, Phys. Rev. D59 (1999) 025001, hep-th/9809065.

[24] R. Kallosh, A. Linde, T. Ortin, A. Peet and A. Van Proeyen, Supersymmetry as a cosmic censor, Phys. Rev. D46 (1992) 5278, hep-th/9205027.

[25] M. Cvetič, D. Youm, Singular BPS saturated states and enhanced symmetries of fourdimensional $N=4$ supersymmetric string vacua, Phys. Lett. B359 (1995) 87, hepth/9507160.

[26] M. Cvetič, D. Youm, Dyonic BPS saturated black holes of heterotic string on a six torus, Phys. Rev. D53 (1996) 584, hep-th/9507090.

[27] M. Cvetič, H. Lü and C.N. Pope, Decoupling limit, lens spaces and Taub-NUT: D= 4 black hole microscopics from $D=5$ black holes, Nucl. Phys. B549 (1999) 194, hepth/9811107.

[28] N. Seiberg and E. Witten, Comment on string dynamics in six dimensions, Nucl. Phys. B471 (1996) 121, hep-th/9603003.

[29] D.Z. Freedman and J.H. Schwarz, $N=4$ supergravity with local $S U(2) \times S U(2)$ invariance, Nucl. Phys. B137 (1978) 333.

[30] M. Cvetič, H, Lü and C.N. Pope, Spacetimes of boosted p-branes and CFT in infinite momentum frame, Nucl. Phys. B545 (1999) 309, hep-th/9810123. 
[31] M. Banados, C. Teitelboim and J. Zanelli, The black hole in three dimensional space time, Phys. Rev. Lett. 69 (1992) 1849, hep-th/9204099. 